\title{
Segmentation of Ultrasonic Images Based on Modified Chan-Vese algorithm
}

\author{
Liqun Wang, ${ }^{1}{ }^{*}$ and Honghui Fan ${ }^{2}$ \\ ${ }^{1}$ School of Information Engineering, Northeast Dianli University, Jilin, Jilin, 132012, China \\ ${ }^{2}$ Computer Engineering School, Jiangsu University of Technology, Changzhou Jiangsu, 213001, \\ China \\ aguoshuqiang@gamil.com
}

Keywords: Ultrasonic image segmentation; Chan-Vese model; Level set method; Image process

\begin{abstract}
The primary ultrasonic image segmentation goal is to partition a given ultrasonic image into different regions representing anatomical structures. Ultrasonic image segmentation is very important because the accurate representation of object and background fluid provides a way to identify many disease. In this paper, a modified Chan-Vese model is proposed for image segmentation, which is based on the similarity between each point and center point in the neighborhood. This model can capture the details of local region to realize the image segmentation in gray-level heterogeneous area. Experimental results show that this method can segment the ultrasonic image with high accuracy, adapt ability and more stable performance compared with the traditional Chan-Vese model.
\end{abstract}

\section{Introduction}

Image segmentation is a key step from image processing to image analysis. It is the basis for expressing the image, and it has an important influence on the measurement of image feature. On the other hand, image segmentation, feature extraction and parameter measurement based on image segmentation can transform the original image to more abstract and more compact forms. It makes it possible to analyze and understanding the image. Image segmentation has attracted the attention of the scholars and researchers since 1970's. So far, thousands of segmentation algorithms have been developed. The existing segmentation algorithms are so many that many classification methods are put forward. Traditional image segmentation methods can generally be divided into the following four classes: (1) threshold-based method [1-2]; (2) edge-based method [3]; (3) region-based method [4-5]; (4) specific theory based method[6].

With the development of artificial neural network, fuzzy set theory and graph theory, some novel segmentation algorithms are proposed combined with these theories [7-8]. Image segmentation based on partial differential equations is an important branch in the field of image segmentation [9-10]. Partial differential equation for image segmentation is a nonlinear method produced in 1980's. This method uses the dynamic model to force the initial curve to the target contour under combined action of internal force and external force. A major advantage of this method is that the curve always can maintain the continuity and smoothness in the process of evolution, and it can achieve the goal of continuous, closed contour extraction. The traditional segmentation methods cannot directly realize this goal. Partial differential equation based segmentation method as a new and effective method of image segmentation provides a good way to solve the problem of the traditional image segmentation.

Chan-Vese is one of the PDE models. The essence of Chan-Vese model is a two-value piecewise constant function (the one is target object, the other is background). Because most images are not simple binary image, in a general image, there are local changes and noise inevitably. Furthermore, such image would cause the convergence rate of Chan-Vese model to become slow. It is necessary to change the curve length coefficient when the Chan-Vese model is applied to noisy image, and the selecting coefficient is difficult to control. If coefficient is too large, the curve will disappear. If the coefficient too small, the curve becomes so small that the correct segmentation would cannot be obtained. Especially, when the traditional Chan-Vese model is used in ultrasonic image and medical 
image, a satisfactory segmentation result cannot be got because of the noise, uneven gray and complex background.

In this paper, a novel Chan-Vese model combined with the neighborhood information is proposed. This model can capture the regional details of ultrasonic image by the method of using the neighborhood information of each point in the image and computing its similarity with the center point to guide the curve to target contour. The experimental results show that this method can realize the segmentation of gray-level heterogeneity ultrasonic image and can reduce the influence of noise to the traditional Chan-Vese model.

\section{The Modified Chan-Vese Model}

The Chan-Vese model is the curve evolution implementation of a piecewise-constant case of the Mumford-Shah model. The basic idea of Mumford-Shah model is to find the optimum relation of energy function for a given image. In the given image , the energy functional is defined as:

$$
E^{M S}(u, c)=\int_{\Omega}(u-I)^{2} d x d y+\mu \int_{\Omega \backslash C}|\nabla u|^{2} d x d y+v|C|
$$

Where $\mu$ and $v$ are positive constants, $u$ is a nearly piecewise smooth approximation of the image $I$, $C$ is the smooth and closed segmenting curve, $\Omega$ denotes the image domain, $\Omega \backslash C$ is the image region not containing curve $C$, and $|C|$ is the length of curve $|C|$. The segmentation image is obtained by minimizing the energy functional $E^{M S}$.

Mumford-Shah model is an ideal model of image segmentation, but in its expression there are both the length item and the area item, it is difficult to solute the equation. Therefore, many scholars put forward the simplified model of the Mumford-Shah. There are two schools to simplify Mumford-Shah model based on elliptic approximation proposed by Ambrosio. The other is c based on the combination of level set methods and Mumford Shah model.

In the Chan-Vese model, the curve evolution $C$ is the boundary of in the definition domain $\omega \subset \Omega$. This assumes that original image $u$ is divided into the internal and external areas by a closed active contour $C_{0}$, and then the energy functional can be defined as:

$$
F(C)=\lambda_{1} \int_{\text {inside }(C)}\left|u(x, y)-c_{1}\right|^{2} d x d y+\lambda_{2} \int_{\text {outside }(C)}\left|u(x, y)-c_{2}\right|^{2} d x d y
$$

The Chan-Vese model uses the global information of the image, so the result of image segmentation is the globally optimal solution. Because the speed function of Chan-Vese model is not correlated with and image gradient, it can be applied to the image whose gradient are both significant and insignificant, and it is suitable for the image whose boundary is smooth or discontinuous. In addition, because the definition of speed function is based on level set, it can automatically detect cavity area of the image.

Usually, the Chan-Vese model assumes that the image is segmented into two homogeneous regions, namely the two constants $C_{1}$ and $C_{2}$ to represent the average gray level of two areas, so this model is also known as Piecewise Constant model. The Chan-Vese model considers global features rather than local feature of the image. Because the adjacent pixels of the image tend to have similar gray level, this paper presents a Modified Chan-Vese model based on neighborhood information. In the Chan-Vese model, the $C_{1}, C_{2}$ respectively represent the average gray-value of inside and outside region, so this model ignores the local characteristics of the image. The modified Chan-Vese model puts the focus on the each point of small neighborhood, and the energy function of the point in neighborhood is defined as:

$$
\begin{aligned}
F_{x}\left(C, f_{1}(x), f_{2}(x)\right)= & \lambda_{1} \int_{\Omega_{1}} g(x-y)\left|I(y)-f_{1}(x)\right|^{2} d y+ \\
& \lambda_{2} \int_{\Omega_{2}} g(x-y)\left|I(y)-f_{2}(x)\right|^{2} d y
\end{aligned}
$$

Where $\mathrm{x}$ is the center point of the small neighborhood, $f_{l}(x)$ is the mean gray-value of homogeneous class with the center point $x, f_{2}(x)$ is the mean gray-value of inhomogeneous class with the center point $x, I(y)$ is the gray-value of the each point in the neighborhood around $x, g()$ is the 
weight coefficient, it means the distance between the center $\mathrm{x}$ and the point in neighborhood, and defined as:

$$
g(x)=\frac{1}{\varepsilon^{2}+k x^{2}} \cdot \frac{N u m(x)}{N}
$$

Where $\varepsilon$ and $k$ are constant coefficient, in this paper, $\varepsilon=1, k=1, N$ is the total of the pixels in small neighborhood, $\operatorname{Num}(x)$ is the total of the pixels in homogeneous regions with the center point $x$.

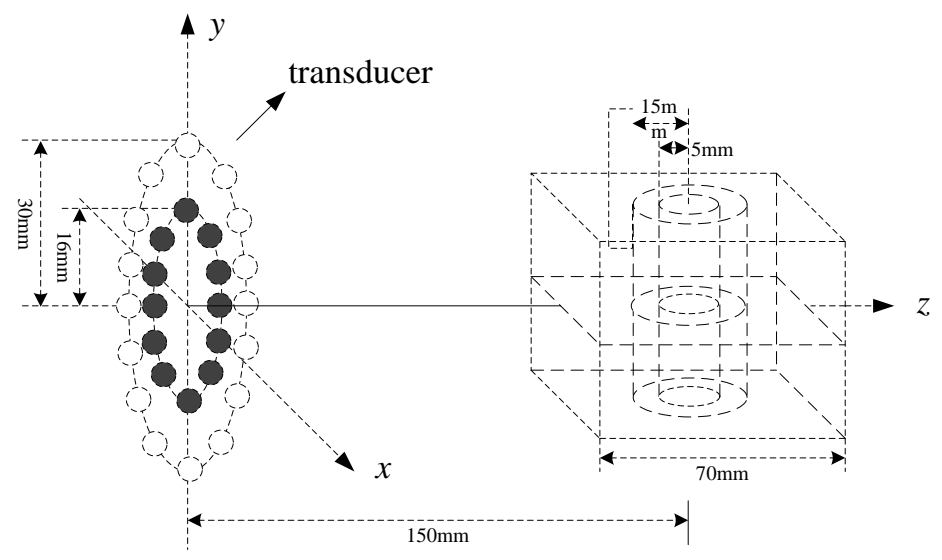

Figure 1. The coordinates of the experimental environment.

(a)

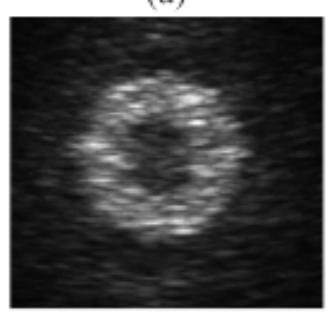

(e)

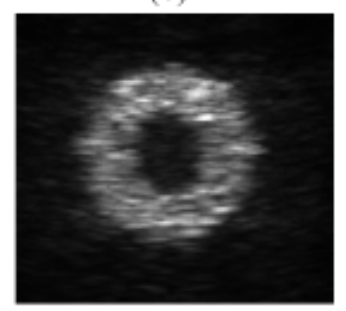

(i)

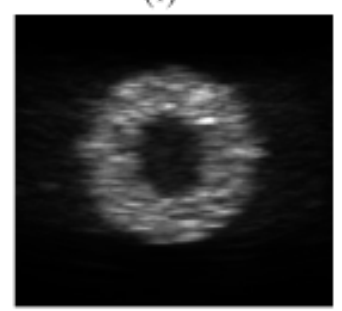

(b)

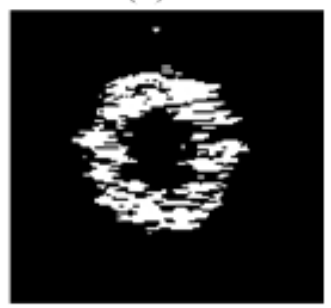

(f)

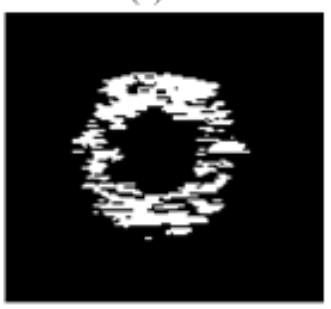

(j)



(c)

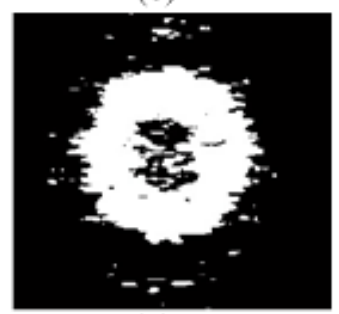

(g)

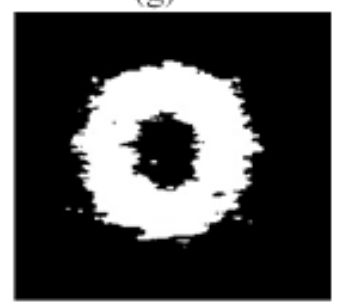

(k)



(d)



(h)

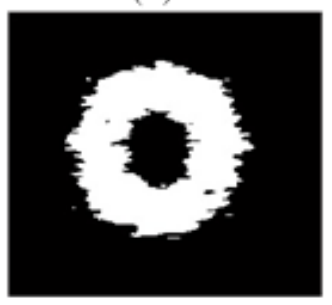

(1)

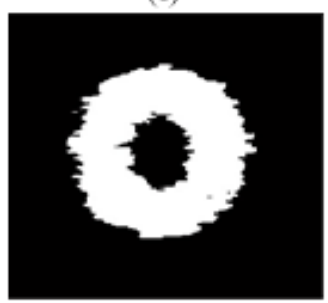

Figure 2. Segmentation result of ultrasonic images using Otu method, traditional Chan-Vese model and modified Chan-Vese model: (a) original ultrasonic imge with 8 shot; (e) original ultrasonic imge with 16 shot; (i) original ultrasonic imge with 32 shot; (b) (f) (j) segmentation result using Otu; (c) (g) (k) segmentation result using traditional Chan-Vese model; (d) (h) (l) segmentation result using modified Chan-Vese model. 


\section{Experimental Results}

In this experiment, a marker is embedded in the test image as shown in Fig. 1 by Matlab development tools. The size of the test image is 512pixel $\times 512$ pixel, and the spatial frequency of embedded marker is $1 / 16$ [cycle/pixel]. In another words, in the test image there are 32 sine waves in $\mathrm{x}$ direction and $\mathrm{y}$ direction respectively. Then we simulate the rotation of the image in $\mathrm{x}, \mathrm{y}, \mathrm{z}$ direction using the parallel projection method. We assume that the focal distance is $18 \mathrm{~mm}$, and the object distance is $600 \mathrm{~mm}$. Then the rotation angle can be estimated by frequency domain analysis as mentioned previously.

In order to verify the validity of the modified Chan-Vese model, three ultrasonic images are segmented by this model, and these ultrasonic images are provided by Tamura laboratory in Yamagata University of Japan. Tamura laboratory have been developing an imaging system adopting the combination of coded excitation and synthetic aperture focusing technique. Using the method, the three-dimensional image was obtained with a very high frame rate and a high spatial resolution. In this encoding transmission method, all transmitters are simultaneously driven and received all signals in $5 \mathrm{~ms}$. One three-dimensional image can be reconstructed by one shot. And the quality of the ultrasonic image can be improved with the increase of the shot counts.

In this paper, a simulation experimental environment is constructed as shown in Fig. 1. In this experimental environment, there is a pipe $1500 \mathrm{~mm}$ away from the transducer array in a sink. The inside diameter and the outside diameter are $8 \mathrm{~mm}$ and $15 \mathrm{~mm}$ respectively. Using coded transmission and multi-element synthetic aperture focusing, three 2-D section ultrasonic images are obtained by 8shot, 16shot and 32shot respectively as shown in Fig. 2(a)(d)(g).

The performance criterion, which is called Segmentation Accuracy (SA), is computed by:

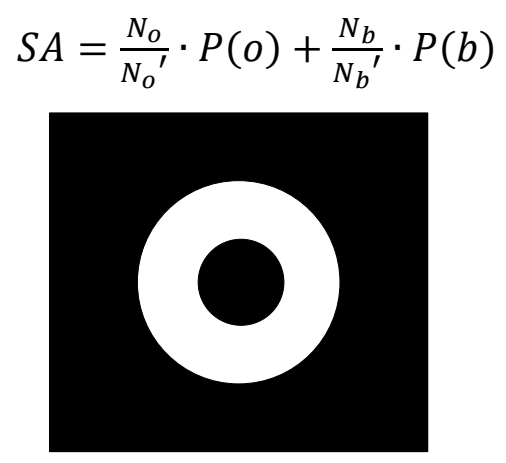

Figure 3. Ideal segmentation result

Where $\mathrm{No}^{\prime}$ and $\mathrm{Nb}^{\prime}$ are the number of pixels in object area and background area respectively in ideal segmentation result, $\mathrm{No}$ and $\mathrm{Nb}$ are the number of pixels in ideal object area and background area in experimental result, $\mathrm{P}(\mathrm{o})$ and $\mathrm{P}(\mathrm{b})$ are the probability of object and background in ideal segmentation result. The ideal segmentation result as shown in Fig. 3, The white area is the pipe in the Fig. 2.(a),(b),(c), and the black area is the background. According to the statistic, No' $=51473$, $\mathrm{Nb}^{\prime}=210671, \mathrm{P}(\mathrm{o})=19.64 \%, \mathrm{P}(\mathrm{b})=80.36 \%$.

Table 1 SA and Processing Time

\begin{tabular}{|c|c|c|c|c|}
\hline & $\begin{array}{l}\text { Otu } \\
\text { Method }\end{array}$ & $\begin{array}{l}\text { Traditional } \\
\text { Chan-Vese } \\
\text { model }\end{array}$ & $\begin{array}{l}\text { Modified } \\
\text { Chan-Vese } \\
\text { model }\end{array}$ \\
\hline \multirow{2}{*}{$\begin{array}{l}32 \\
\text { shot }\end{array}$} & SA & $72.6 \%$ & $89.3 \%$ & $94.2 \%$ \\
\hline & $\begin{array}{l}\text { Processing } \\
\text { time }(\mathrm{ms})\end{array}$ & 4.2 & 123.2 & 154.8 \\
\hline
\end{tabular}

Using the Otu method, traditional Chan-Vese model and modified Chan-Vese model the image segmentation results were obtained as shown in Fig. 2. The segmentation accuracy and processing time is shown in Table 1. The results show that improved Chan-Vese model can segment the ultrasonic image more reasonable in the low SNR environments comparing with the Otu method and 
traditional Chan-Vese model. Especially in the case of 32shot, the modified Chan-Vese model not only gets rid of the influence of noise on image segmentation, but also gets the pipe structure clearly in the ultrasonic image.

\section{Conclusion}

The traditional Chan-Vese model cannot divide non-uniform image, and it is sensitive to noise. In order to solve this problem, we propose a modified Chan-Vese model based on local information. In this method, the similarity between each point and its neighborhood is used, thus the local characteristics of heterogeneous image is maintained. Moreover, because of the use of local image information, this model can segment images with intensity inhomogeneity in fewer iteration. The experimental result shows that the modified Chan-Vese model is effective and robust compared with traditional Chan-Vese model.

\section{References}

[1] J. Kittler and J. Illingworth: Minimum error thresholding, Pattern Recognition, Vol.19 (1986) No.1, p.41 - 47.

[2] Du Feng,S. Wenkang and C. Liangzhou: Infrared image segmentation with 2-D maximum entropy method based on particle swarm optimization (PSO), Pattern Recognition Letters, Vol.26 (2005) No.5, p.597 - 603.

[3] T. Su,M. Yang and T. Wu: Morphological segmentation based on edge detection for sewer pipe defects on CCTV images, Expert Systems with Applications, Vol.38 (2011) No.10, p.13094 13114.

[4] F. Y. Shih and S. Cheng: Automatic seeded region growing for color image segmentation, Image and Vision Computing, Vol.23 (2005) No.10, p.877 - 886.

[5] K. Saeed and M. Albakoor: Region growing based segmentation algorithm for typewritten and handwritten text recognition, Applied Soft Computing, Vol.9 (2009) No.2, p.608 - 617.

[6] M. Vermandel,N. Betrouni and C. Taschner: From MIP image to MRA segmentation using fuzzy set theory, Computerized Medical Imaging and Graphics, Vol.31 (2007) No.3, p.128 - 140.

[7] N. Teimouri,M. Omid and K. Mollazade: A novel artificial neural networks assisted segmentation algorithm for discriminating almond nut and shell from background and shadow, Computers and Electronics in Agriculture, Vol.105 (2014) No.1, p.34 - 43.

[8] N. Narappanawar,B. M. Rao and M. Joshi: Graph theory based segmentation of traced boundary into open and closed sub-sections, Computer Vision and Image Understanding, Vol.115 (2011) No.11, p.1552 - 1558.

[9] A. L. Boyer,C. Cardenas and F. Gibou: Evaluation of a semi-automated segmentation technique using partial differential equations, International Journal of Radiation Oncology Biology Physics, Vol.57 (2003) No.2, Supplement, p.206 - 207.

[10] J. Weickert: Efficient image segmentation using partial differential equations and morphology, Pattern Recognition, Vol.34 (2001) No.9, p.1813 - 1824. 